\title{
Mixed substrates in environmental biotechnology
}

G. Hamer, Th. Egli and M. Snozzi

Institut für Gewässerschutz und Wassertechnologie, Eidgenössische Technische Hochschule, and Eidgenössische Anstalt für Wasserversorgung, Abwasserreinigung und Gewässerschutz, Ubberlandstrasse 133, CH-8600 Dübendorf (Switzerland)

Summary. The scope of environmental biotechnology is defined and four examples of recent research in environmental biotechnology involving various types of mixed substrates are discussed in detail and their probable impacts assessed. The four examples are: multiple carbon energy substrate (pollutant) biodegradation by both mono and mixed cultures, the biodegradation of whole microbial cells, the biodegradation of single compounds satisfying dual physiological requirements, i.e., mixed carbon and mineral nutrient sources, and simultaneous nitrification and denitrification.

Key words. Microbes; environment; biotechnology; process; treatment; biodegradation; mixed substrates; pollutants. 


\section{Introduction}

Environmental biotechnology is the sector of biotechnology that concerns microbial processes for efficient, effective and economic waste treatment, particularly the elimination of noxious pollutants; microbial processes for the remediation of previously polluted natural environments and for cleaning up dirty naturally occurring feedstock; the systematic evaluation and control of microbially mediated degradation processes in engineered environments, and the impact of both intentional and accidental contamination of natural environments with non-indigenous microbes.

Pollutants and materials subject to either degradation or modification are inevitably non-indigenous with respect to the pristine state of the natural environments with which they interact. Pollutants can be either biotic or xenobiotic in nature. Xenobiotic pollutants include both organic and inorganic matter, whilst biotic pollutants are inevitably organic and comprise microbes, active elements thereof and their products. In all engineered environmental processes and systems, the mediating microbes involved are exposed to either a mixture of substrates that can potentially satisfy the same physiological requirement of the microbes, or mixed substrates that can potentially satisfy more than one physiological requirement. Because of the exclusively non-aseptic operation of all environmental biotechnological processes, process cultures are inevitably mixtures of microbes functioning either in concert or in conflict with each other. Additionally, process environments are frequently lean with respect to substrate availability and are subject to marked physical and chemical heterogeneities.

Traditional approaches to microbial physiology that form the basis for the understanding of processes involving microbes generally fail to recognize that microbes functioning in engineered environments are subjected to either some or all of the above-mentioned constraints, and that in real processes the roles of surfaces and interfaces are frequently dominant. In addition, substrates are frequently neither water-soluble nor water-miscible to any significant degree. Further, engineered environmental processes rarely involve only a single portion of an elemental cycle; rather they either involve major portions of individual elemental cycles or interactions between elemental cycles as a result of intimate association between heterotrophs and/or autotrophs functioning either in their respective modes or, in the case of the latter, mixotrophically.

In this short review, attention will be focussed on four areas of study concerning mixed and multiple substrate utilization with which the authors' laboratory has been involved. The potential impacts of advances made are also discussed with respect to both waste treatment processes and the possibilities that exist for better control of microbially mediated processes that adversely affect the operation of engineered systems in natural environments.

\section{Multiple carbonaceous substrate (pollutant) biodegradation}

In engineered environments microbes frequently grow in the presence of a diversity of functionally similar substrates. The pattern of utilization of multiple substrates by monocultures is dependent on the concentrations of the substrates. When diverse mixed cultures are involved, utilization patterns are usually dependent on culture composition. Mixed cultures are of two distinct types, i.e., cultures that contain more than $90 \%$ of the primary carbon energy substrate utilizer together with a small number of satellite strains that support its effective functioning, and cultures that exhibit true diversity, with many component strains that are able to utilize the broad spectrum of substrates available. In most respects, mixed cultures of the former type behave in a very similar manner to pure monocultures that utilize the same primary substrate, but are frequently less fastidious. Mixed cultures that have been repeatedly grown on single carbon energy substrates in the laboratory can be expected to be of the former type.

The study of both mono and mixed cultures growing on a diversity of carbon energy substrates has been subject to a remarkable degree of neglect. As far as the batch growth of the former is concerned, the concepts proposed by Monod nearly fifty years ago ${ }^{18}$ have remained essentially unchallenged and the kinetics involve summation of those applicable for single substrate utilization by the same microbe. Similar behavior has also been reported for mixed cultures in laboratory studies ${ }^{25}$. In the case of dual substrates, the question of substrate concentration has recently become a consideration during both batch and chemostat cultures, and it has been shown that microbes that are able to utilize a spectrum of available substrates are more successful in competition ${ }^{10}$.

Perhaps even more dramatic than the results concerned with competition were results obtained for the growth of the methylotrophic yeast, Hansenula polymorpha, in dual substrate chemostat culture ${ }^{8}$. Here, not only were the two substrates, glucose and methanol, utilized simultaneously, but in the case of dual substrate mixtures, methanol could be completely utilized at dilution rates, i.e., imposed growth rate constants, exceeding the critical dilution rate for wash-out when methanol was supplied as the sole substrate. Further, this phenomenon was dependent on the substrate mixture ratio. The results are shown in figure 1. Recently, in the case of very low concentrations of certain dual component sugar mixtures, simultaneous utilization by Escherichia coli in batch cultures has been demonstrated ${ }^{24}$, although at higher concentrations cultures with the same substrates exhibit diauxic batch growth. This suggests that at low substrate concentrations simultaneous utilization is probably the rule rather than the exception.

In both sewage and industrial wastewater treatment it is the accepted practice to describe the composition of 


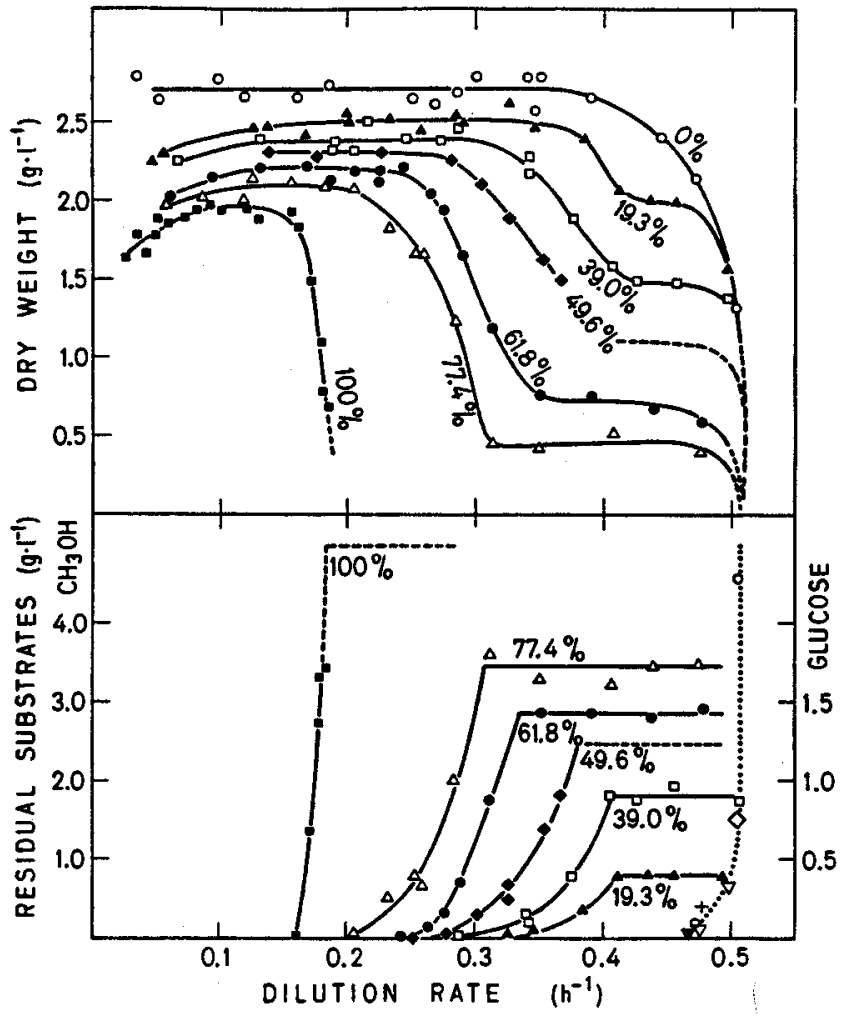

Figure 1. Dry weight of cells formed and residual concentrations of carbon substrates during growth of Hansenula polymorpha CBS 4732 in a chemostat as a function of the composition of the methanol $\left(C_{1}\right) / g l u-$ $\operatorname{cose}\left(\mathrm{C}_{6}\right)$-mixture supplied in the inflowing medium. The composition of the methanol/glucose-mixture is given in weight percent. For clarity, only the percent methanol is used to characterize mixture compositions. The total concentration of substrate (methanol + glucose) in the feed was always $5.0 \mathrm{~g} \cdot \mathrm{l}^{-1}$. The symbols for residual glucose are: $0,0 \% \mathrm{C}_{1}$; $\triangle, 19.3 \% \mathrm{C}_{1} ; \square, 39.0 \% \mathrm{C}_{1} ; \bullet, 49.6 \% \mathrm{C}_{1} ; \bullet, 61.8 \% \mathrm{C}_{1} ; \Delta, 77.4 \% \mathrm{C}_{1}$.

the process feed in terms of 'lumped' parameters such as chemical oxygen demand (COD), dissolved organic carbon (DOC), etc. Clearly, the use of such 'lumped' parameters is unavoidable in the case of municipal sewage with its highly complex composition, but such an approach is not necessarily appropriate when considering some industrial wastewater streams. For example, petrochemical industry wastewater streams frequently contain less than 100 organic pollutants, with more than $80 \%$ of the pollutant load being represented by less than 10 key compounds, so that studies concerning bulk carbonaceous pollutant elimination from such wastewater streams can frequently concentrate on the key pollutants present ${ }^{11}$. Such an approach was reported for a wastewater stream containing methanol, phenol, acetone and isopropanol as key pollutants ${ }^{29}$, where the selected mesophilic mixed culture was shown to comprise three distinct moieties which utilized methanol, phenol and acetone, respectively, as their growth substrates. Isopropanol was eliminated by co-metabolic activity of the acetone-utilizing moiety, but in spite of this, interactions between moieties were negligible, so that neutralism occurred. Clearly, such a single study was inadequate for the establishment of generally applicable concepts concerning the aerobic biodegradation of multiple carbonaceous pollutants.

As a result, an investigation was undertaken of the aerobic biodegradation of a mixture of solvents, which had been shown, individually, to be biodegradable, comprising methanol, methylene chloride, acetone and isopropanol ${ }^{3}$. The reasons for this study were the widespread utilization of these solvents, frequently in association with each other, and their mode of use which often results in waste rather than recovery. An example of such wastage is the use of isopropanol for airport apron de-icing, and hence its intermittent presence in significant concentrations in apron drainage, which should be subject to collection and treatment prior to discharge.

Continuous flow enrichment studies with the solvent mixture as carbon energy substrate resulted in a mixed culture of restricted complexity consisting of only two primary substrate degrading strains and two satellite strains, representing ca $1 \%$ of the culture, that utilized none of the supplied substrates. One of the primary substrate degrading strains was a non-methylotroph which formed $60 \%$ of the enrichment culture and did not grow on either methanol or methylene chloride; the other was a facultative methylotroph, representing $39 \%$ of the enrichment culture, which failed to grow on methylene chloride, acetone or isopropanol. However, in spite of this, when either the crude enrichment culture or a reconstituted total mixed culture was grown on a mixture of all four solvents, methylene chloride was biodegraded as evidenced by chemostat culture data for these two cultures, shown in figure 2 , where no methylene chloride was present in the culture supernatants until the critical dilution rates were approached. Presumably, cometabolism was in some way responsible for methylene chloride elimination. Of further significance as far as wastewater treatment is concerned was that markedly reduced biomass concentrations were observed at lower dilution rates, suggesting enhanced endogenous and/or exogenous activity and the build-up of acetone in culture supernatants as critical dilution rates were approached. When the reconstituted binary culture was grown with only isopropanol and methanol as supplied carbon energy substrates, acetone accumulation was again observed prior to the detection of any isopropanol in the culture supernatant. When the non-methylotroph was grown alone with either isopropanol or acetone as its sole carbon energy substrate, acetone accumulation occurred in both cases, but in the latter case was also accompanied by an accumulation of methanol as critical dilution rates were approached. The behavior patterns for all three culture systems are shown in figure 3.

In order to further elucidate the role of the 'apparently' non-methylotrophic bacterium both in the biooxidation of acetone and isopropanol and in methanol accumulation, the bacterium was grown in chemostat culture at an 


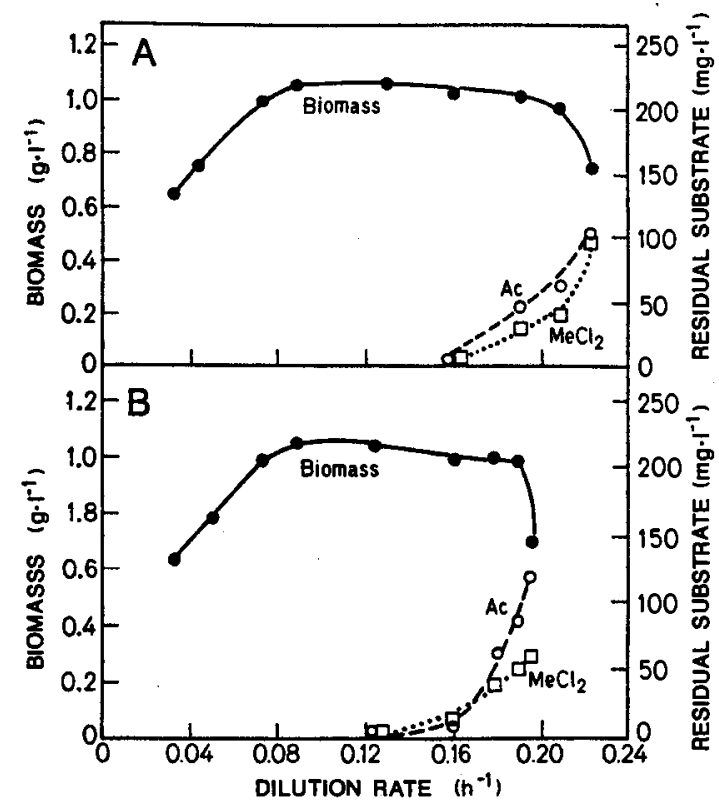

Figure 2. Dry weight of cells (biomass) formed and residual substrate accumulated during chemostat cultures of $A$ the undefined enrichment culture and $B$ the defined two-component mixed culture, on methanol, $0.8 \mathrm{~g} \cdot 1^{-1}$, acetone ( $\mathrm{Ac}$ ), $0.4 \mathrm{~g} \cdot 1^{-1}$, isopropanol, $0.4 \mathrm{~g} \cdot \mathrm{1}^{-1}$, and methylene chloride $\left(\mathrm{MeCl}_{2}\right), 0.8 \mathrm{~g} \cdot 1^{-1}$, at various dilution rates.

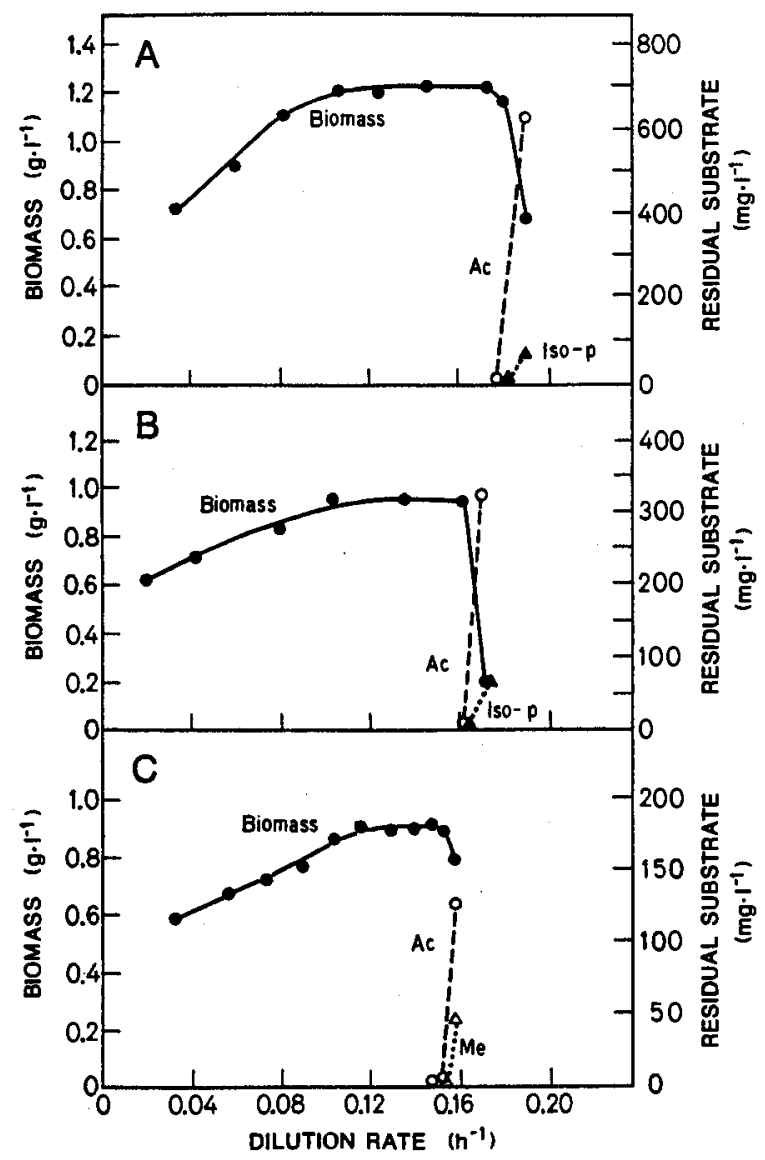

Figure 3. Dry weight of cells (biomass) formed and residual substrate accumulated during chemostat cultures of $A$ the defined two-component mixed culture on methanol (Me) $1.0 \mathrm{~g} \cdot 1^{-1}$, and isopropanol (Iso-p) $1.0 \mathrm{~g} \cdot 1^{-1}, B$ the non-methylotroph on isopropanol, $1.2 \mathrm{~g} \cdot 1^{-1}$, and $C$ the non-methylotroph on acetone $(A C) 1.2 \mathrm{~g}^{\cdot-1}$, at various dilution rates.

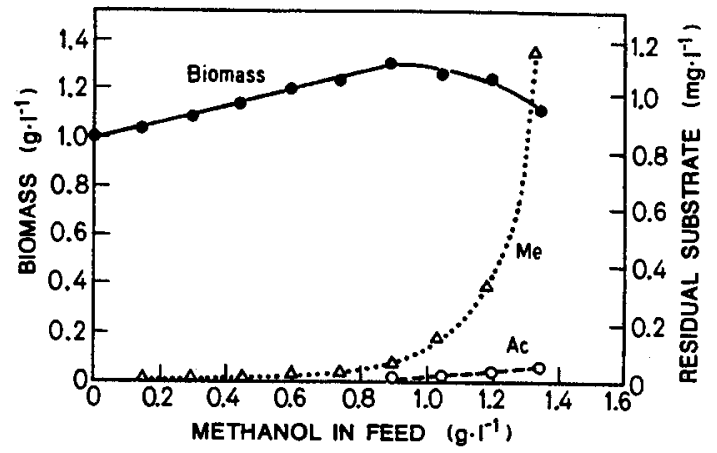

Figure 4. Dry weight of cells (biomass) formed and substrate accumulated during chemostat culture of the non-methylotroph on mixtures of isopropanol, $1.2 \mathrm{~g} \cdot 1^{-1}$ and methanol, as indicated, at a constant dilution rate of $0.12 \mathrm{~h}^{-1} . \mathrm{Ac}=$ acetone.

intermediate dilution rate, with a constant concentration of isopropanol but a range of concentrations of methanol present in the chemostat feed. At lower methanol:isopropanol ratios, all of the isopropanol and a major fraction of the methanol were eliminated, but when the weight fraction of methanol in the feed approached $50 \%$ by weight, methanol accumulated to concentrations which indicated that there was no methanol elimination, i.e., washout occurred. Further, as methanol accumulation became more pronounced, an accumulation of low concentrations of acetone also occurred. These results, which further emphasize the complexities of co-metabolic activity, are illustrated in figure 4 . Other results from batch cultures ${ }^{3}$, not illustrated here, suggest that the 'apparently' non-methylotrophic bacterium first converts isopropanol into acetone and methanol prior to growth. Further, experimental results with substrate mixtures comprising either acetone or isopropanol and ${ }^{14} \mathrm{C}$ labeled methanol indicated that the methanol carbon was completely converted to $\mathrm{CO}_{2}$ so that methanol acted only as an energy source, a hypothesis that was supported by the detection of methanol-dissimilating enzymes in this bacterium.

The investigations discussed so far have all been carried out at mesophilic growth temperatures, but it has been suggested that, in the case of those industrial wastewaters that are produced at elevated temperatures, biotreatment at elevated temperatures should be investigated ${ }^{31}$. In this respect, the application of thermotolerant and thermophilic bacteria for the biotreatment of model petrochemical industry wastewaters has recently been investigated $^{2}$. In one part of this study, the effectiveness of a binary mixed culture comprising a thermotolerant methylotrophic bacterium and a thermophilic nonmethylotrophic solvent-utilizing bacterium in biooxidizing a substrate mixture consisting of methanol, ethanol and isopropanol in the thermotolerant temperature range for growth was investigated. Again, acetone formation from isopropanol, this time under transient-state continuous flow operating conditions, manifested itself. 
The question of an unexpected product build-up in defined mixed cultures growing on multiple carbon energy sources clearly requires elucidation if any sensible understanding of such systems is to be established.

\section{Microbial biodegradation of solids}

The widespread introduction of effective municipal sewage and industrial wastewater treatment since 1970 has resulted in a much increased production of the main by-product, waste sludge. In Switzerland, waste sewage sludge production was estimated to be 250,000 tons per annum on a dry basis in 1985 , rising to 300,000 tons per annum on the same basis by $2000^{1}$. The major fraction of the carbonaceous biodegradable pollutants present in waste sewage sludge consists of essentially water-insoluble aggregated solid matter, whilst in settled municipal sewage, the feed for the vast majority of activated sludge secondary treatment processes, some $20-40 \%$ of the biodegradable carbonaceous pollutant load is in the form of discretely dispersed suspended solids. In both cases, the solid matter comprises both microbial and non-microbial solids, although the former predominante in waste sludge. For effective sludge and/or sewage treatment it is essential to understand the solubilization/ biodegradation of particulate solid matter, and in order to achieve this objective it is essential to differentiate between the two categories of solids mentioned above. Microbes consist of a complex, essentially fluid cytoplasm that is surrounded by a rigid cell wall and membrane which maintain the physical and chemical in tegrity of the microbial cell. Non-microbial solids are generally either continuous or porous solids.

In both sewage and waste sludge biotreatment process feeds, non-microbial biodegradable solids can be typified by cellulose particles, the microbial solubilization/ biodegradation of which has been extensively studied and described in terms of a complex shrinking-site model which ultimately predicts a modified cube root law type of pattern ${ }^{13}$. In the case of microbial solids, overall application of the cube root law is clearly inapplicable except to cell wall fragments, because hydrolysis of whole microbial cells involves puncturing and/or bursting of the cell wall/membrane and release of the cytoplasm, which produces both soluble matter and cell wall fragments ${ }^{17}$.

With respect to their physical state, microbial cells clearly represent a relatively unfavorable substrate for microbial growth. However, in other respects, such as their mineral composition and overall carbon and energy contents, they are ideal substrates for aerobic, heterotrophic growth, exhibiting neither energy deficiency nor energy excess provided all of their components can be utilized simultaneously. This can be illustrated by considering the relationship that exists between the maximum observed biomass yield coefficient for aerobic microbial growth on particular carbon energy substrates, expressed in terms of the gram of dry microbial biomass produced per gram of suivstrate carbon utilized, and the heat of combustion of the substrate expressed in kilocalories per gram of substrate carbon ${ }^{16}$. Potential carbon energy substrates fall either into the energy-deficient category, where the maximum observed yield coefficient increases proportionally with the substrate's energy content, or into the energy excess category, where the maximum observed yield coefficient remains constant, irrespective of the substrate's energy content. Data concerning the heat of combustion of microbial cells ${ }^{20}$ indicate that as carbon energy substrates they fall at the intersection the two substrate categories; the theoretical maximum yield coefficient for microbial biomass production from microbial biomass is $0.67 \mathrm{~g} \cdot \mathrm{g}^{-1}$ on a whole cell basis.

In all biotreatment processes involving whole microbial cells in the process feed, it is important to differentiate between the process microbes which essentially grow, but are also subject to death/lysis and 'cryptic' growth mechanisms, on the one hand, and the supposedly non-growing feed (substrate) microbes, which are only subject to the degradative processes mentioned above, on the other hand. Traditional treatment technology for waste sewage sludge involves mesophilic anaerobic digestion, but more recently aerobic thermophilic treatment of sludge either as an independent process or as a pretreatment process prior to traditional treatment has become increasingly more widespread ${ }^{12}$. A schematic diagram for the aerobic thermophilic process for the biodegradation of microbial cells is shown in figure 5 . Although in a completely aerobic biodegradation process, extensive carboxylic acid formation would not be expected, the heterogeneity that occurs in processes with a high solids content leads to restricted oxygen availability. As a result the facultative anaerobic bacteria functioning within the overall process culture will promote carboxylic acid formation.

Aerobic thermophilic waste sewage sludge treatment processes can be operated in either the continuous flow mode or semi-continuously, although when hygienization is a processing prerequisite, semi-continuous operating procedures that eliminate bypassing within the bioreactor are preferred ${ }^{12}$. Because of this, the semi-continuous operation of a non-defined mixed culture thermophilic process utilizing microbial cells as the substrate was investigated ${ }^{17}$. Some of the results obtained are shown in figure 6, where a concentrated suspension of baker's yeast was used as a model process feed. During the operating cycle neither the $\mathrm{pH}$ nor the dissolved oxygen concentration were controlled and, in the case of the latter, oxygen availability to the processes was restricted, never exceeding $5 \%$ of saturation with air at $60^{\circ} \mathrm{C}$. The process of feed organism solubilization was indicated by the progressive reduction of suspended solids, both on a total dry weight (TSS) and on a volatile (ash-free) dry weight (VSS) basis, with corresponding production of soluble organic compounds. During the later part of the 


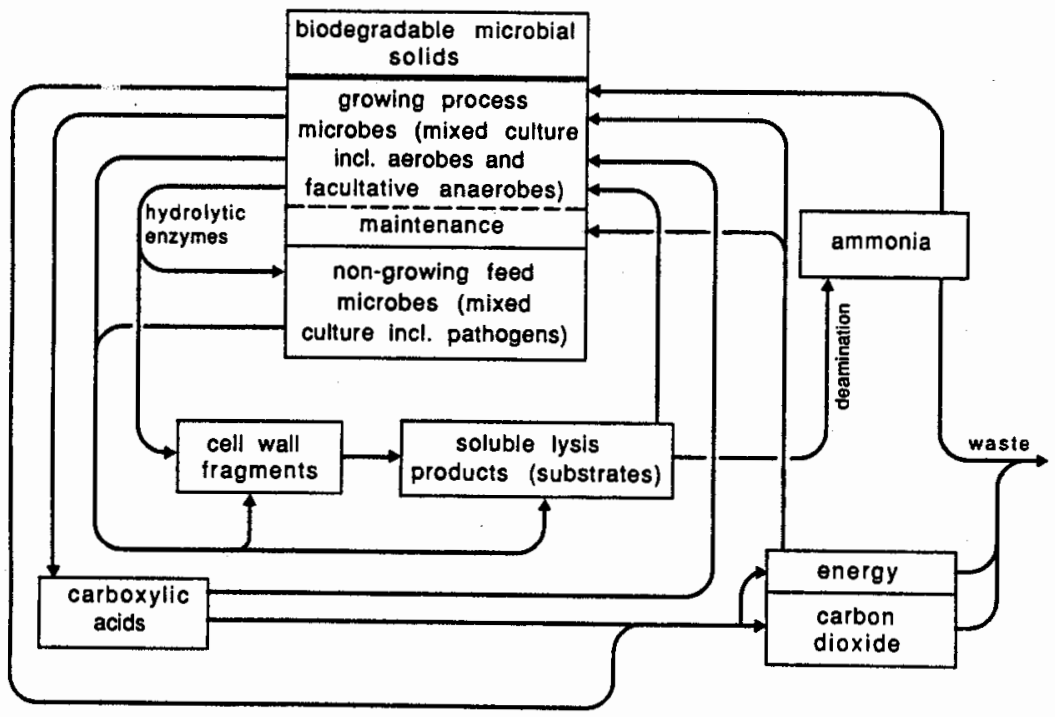

Figure 5. Schematic diagram for the aerobic thermophilic biodegradation of microbial cells.

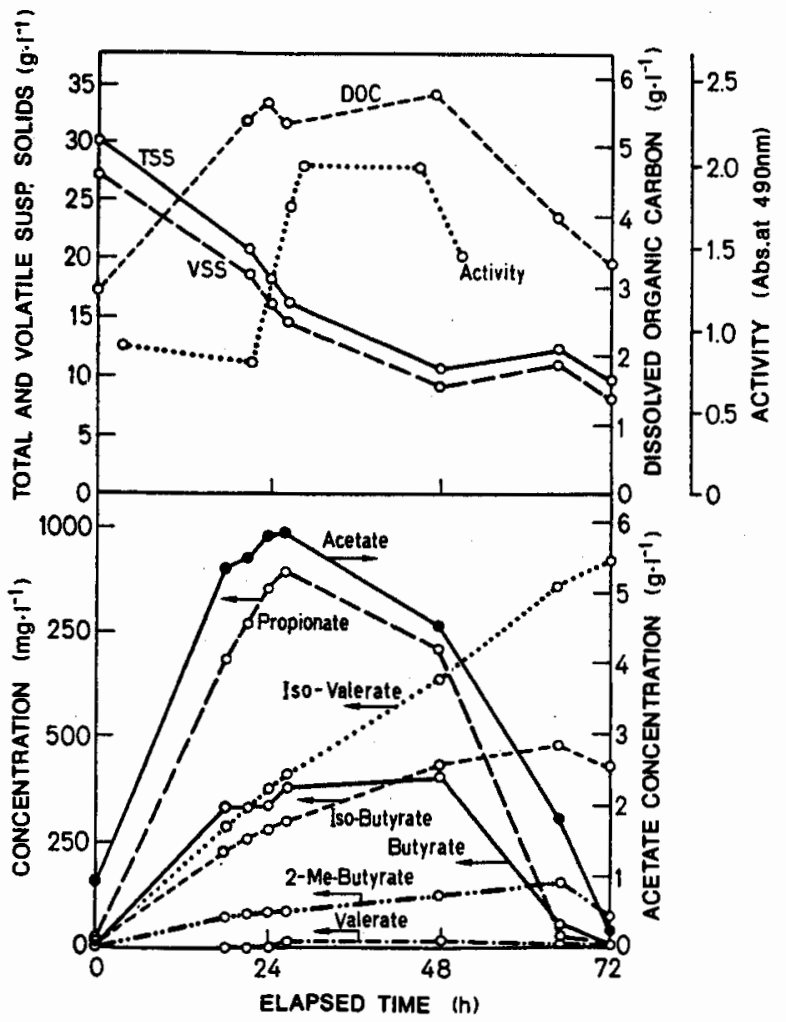

Figure 6. Changes in the concentrations of total suspended solids (TSS), volatile suspended solids (VSS), dissolved organic carbon (DOC), and low molecular weight carboxylic acids and dehydrogenase activity (acitivity) measure by formazan formation in a test with iodonitrotetrazolium chloride during the semi-continuous aerobic thermophilic biodegradation of microbial solids (yeast cells) with respect to elapsed time.

operating cycle, the soluble organic compounds declined as they were utilized by the process culture. A significant fraction of the soluble organic compounds produced were volatile carboxylic acids, particularly acetic acid.
The dehydrogenase activity of the process culture also underwent a corresponding increase and decline.

Of concern in all waste treatment processes handling solid biodegradable matter is the degree of completeness of solids elimination that can be attained within realistic time and economic frameworks. It is clearly evident from figure 6 , that during a cycle time of $72 \mathrm{~h}$ the VSS concentration was only reduced to ca $30 \%$ of the original value, indicating a considerable residual fraction of potentially biodegradable matter. This is a major disadvantage if such solids degradation processes are to be employed alone rather than in sequence with an anaerobic digestion step. Further, the residual concentrations of the particularly odoriferous carboxylic acids that were produced, but not fully degraded, must also be considered in the same context. Clearly, a significant fraction of the residual volatile suspended solids comprises process, rather than feed, microbes, but even so, when effective mineralization is the overall process objective, marked reductions must be achieved in the concentration of residual biodegradable solids. In this latter respect, considerable possibilities exist, particularly minimization of the yield coefficients of the process microbes by enhancing both endogenous and exogenous activities or in uncoupling growth and respiration, particularly with respect to the facultative anaerobic fraction of the process mixed culture.

\section{Compounds satisfying multiple physiological requirements}

The biodegradation of compounds containing both carbon and another major nutrient for the responsible microbe has not been widely studied, especially the consequences of growth in the presence of other compounds capable of satisfying the second requirement. In most respects it is xenobiotic compounds rather than naturally 
occurring compounds such as amino and nucleic acids that are of primary concern.

An extensive range of organic compunds containing nitrogen, sulphur and/or phosphorus in addition to carbon have been produced. Some of the best known as far as biodegradability studies are concerned are compounds such as the s-triazines, sulphonated hydrocarbons, organophosphates and azo dyes ${ }^{15}$. Frequently, the xenobiotic organic chemical in question behaves primarily as the source of a nutrient other than carbon. In the presence of other, more easily utilizable sources of such nutrients, many xenobiotics are apparently recalcitrant.

In our laboratory, the biodegradation of aminopolycarboxylic acids, particularly nitrilotriacetic acid (NTA), a chelating agent that is widely incorporated into household washing powders, is of major interest. In Switzerland alone several thousand tons of NTA are discharged directly into the sewers and, therefore, it is important that NTA should be amenable to biodegradation under the conditions pertaining in municipal sewage treatment plants. NTA elimination depends on acclimation of the process culture mediating the treatment process, and removal efficiency depends on numerous factors such as NTA concentration, hydraulic and sludge residence times, temperature, loading, water hardness and heavy metal concentrations. In general, emphasis has been placed on the chemodynamics of NTA disappearance and microbiological factors have been neglected.

Even so, several gram-negative and a gram-positive aerobic NTA-degrading bacteria have been isolated in pure culture during the past 20 years, but only recently have some of these bacteria been subject to detailed study concerning their taxonomic position ${ }^{9,26}$. Whereas previously gram-negative isolates were ascribed to the genus Pseudomonas, they would now seem to be more appropriately included in the $\alpha$-subgroup of the purple photosynthetic bacteria. The taxonomic position of the one gram-positive isolate investigated still remains uncertain. In addition to the aerobic NTA-degrading bacteria, mixed cultures of facultatively anaerobic bacteria that are capable of NTA-degradation under anoxic denitrifying conditions have been enriched, and recently a pure strain of one such bacterium has been isolated ?

A pathway for the aerobic biodegradation of NTA was proposed by Cripps and Noble ${ }^{4}$. The initial step in the pathway involved a soluble NTA-monooxygenase which converted NTA into iminodiacetate and glyoxylate. However, subsequent steps in the proposed pathway remain less clear and the involvement of glycine remains to be confirmed. Much of the difficulty in elucidating the pathway has stemmed from the difficulties of analyzing either NTA or iminodiacetate in cell free extracts, but it is pleasing to report that these problems have now been resolved by the application of ion exclusion and ion chromatography, respectively ${ }^{23}$. A first outcome of the availability of these novel analytical procedures was nullification of the hypothesis that the NTA-monooxyge- nase was also involved in iminodiacetate breakdown to glycine and glyoxylate. This clearly indicated the necessary presence of a hitherto unsuspected and still unidentified enzyme ${ }^{23}$. Elucidation of the structure and function of NTA-monooxygenase and identification of the subsequent enzymic steps in the aerobic degradation of NTA are in progress.

Perhaps equally important is the characterization of the enzymes responsible for NTA catabolism in the newly isolated denitrifying strain. Clearly, they must function independently of molecular oxygen and, therefore, a second pathway for NTA-breakdown must exist. There is strong evidence that a dehydrogenase is responsible for the first step in NTA-breakdown under anoxic growth conditions (Kemmler, J. and Wanner, U., unpubl. results), whereas, when the facultative anaerobic NTA degrading bacterium is grown aerobically, NTA-oxidase activity is detectable. Knowledge concerning the distribution of various NTA-degrading bacteria and their relative contributions to NTA degradation in sewage treatment plants is clearly of importance, but a prerequisite for this is an understanding of the regulation and expression of NTA-related enzymes under relevant system conditions.

In addition, it is also important to determine those factors that interfere with the effective functioning of specific pollutant degrading microbes in both simulated and real process environments, and to find out whether the strains studied in the laboratory are, in fact, those of relevance in real systems. Clearly, this is an enormous endeavor. It can already be reported that a number of the strains under study in our laboratory are also found to be functioning in both sewage treatment plants and natural environments, and that significant progress is also being made in studies concerning the interaction of excess ammonia with NTA-biooxidation (Wilberg, E., unpubl. results).

\section{Simultaneous nitrification and denitrification}

Nitrogenous compounds are ubiquitous constituents of municipal sewage and, depending on origin, are also frequently encountered in agricultural and industrial wastewaters. Whilst nitrogen is an essential nutrient for microbial growth and, as such, is consumed during the biotreatment of sewage and other wastewaters, carbon: nitrogen ratios are frequently imbalanced, so that availability markedly exceeds the requirements for microbial growth. The predominant nitrogenous pollutants in sewage are urea and proteins which are hydrolyzed and deaminated, respectively, to form ammonia. Nitrogen is assimilated for microbial growth as ammonia, and when none of the other steps in the nitrogen cycle occur any excess ammonia remains untreated and is discharged. Fortunately, other steps in the nitrogen cycle do occur to varying extents and, depending on both the treatment process feed and the required discharge quali- 
ty, can be exploited. The relevant steps are nitrification and denitrification.

It is frequently assumed that denitrification only occurs in anoxic environments, because the denitrifying capacity of some bacteria is totally inhibited by oxygen. However, some denitrifying bacteria also function as such in oxygen-containing environments ${ }^{22}$. For sewage and wastewater treatment this means that when nitrification is not subject to inhibition by either organic matter or metal ions, simultaneous aerobic organic pollutant biooxidation, nitrification and denitrification can be envisaged within a single aeration basin rather than in the sequential, multi-basin, segregated approach that presently dominates practice.

The concept of aerobic denitrification referred to above $^{22}$ involved studies with Thiospaera pantotropha, which is a facultatively anaerobic, facultatively autotrophic sulphur bacterium ${ }^{21}$ and, hence, a bacterium which clearly could play a role in the sulphur cycle, specifically in sulphide oxidation, given appropriate environmental conditions. Such a bacterium would not necessarily be considered ideal for harnessing in aerobic treatment processes, and denitrifying methylotrophs, in particular Hyphomicrobium spp., offer superior potential for more general application.

Biogas is a well-known by-product from anaerobic digestion processes for waste sewage sludge. As produced, with a methane content of ca $60 \%$ methane, it is not always a readily marketable fuel. This tends to encourage self-utilization of any biogas produced by sewage treatment works, usually for maintaining the operating temperature of the anaerobic digestion process. However, an additional use could be as the supplied substrate for a combined aerobic nitrification/denitrification process.

Ammonia oxidation by obligate methanotrophic bacteria was first reported forty years ago ${ }^{14}$. Subsequent investigations ${ }^{5,19}$ showed the oxidation only proceeded as far as nitrite, the step attributed to Nitrosomonas spp. in conventional sewage treatment processes. However, Drozd et al. ${ }^{6}$ have reported that a Methylococcus sp. was able to oxidize ammonia completely to nitrate, without nitrite accumulation, i.e., it undertook the combined role of both Nitrosomonas and Nitrobacter spp.

In any unprotected process where bacterial growth on methane occurs, the mixed culture responsible will comprise not only obligate methane-utilizing bacteria, but also methylotrophic bacteria, specifically $\mathrm{Hyphomicrobi-}$ um spp., and a range of heterotrophic bacteria. In such mixed cultures, it was shown that the role of the $\mathrm{Hy}$ phomicrobium sp. present was to scavenge methanol produced from methane by the methane-utilizing moiety in the mixed culture ${ }^{30}$. When this same Hyphomicrobium sp. was grown in pure culture at $32^{\circ} \mathrm{C}$ in the presence of ammonium nitrate, denitrification became evident, in terms of an increasing oxygen-based biomass yield coefficient, when the dissolved oxygen concentration in the growth medium fell below ca $25 \%$ of saturation with air at one atmosphere total pressure. On the other hand, when ammonium chloride was substituted for ammonium nitrate, the oxygen-based yield coefficient remained constant ${ }^{27}$. Evaluation of these results showed that denitrification occurred simultaneously with respiration with oxygen, i.e., aerobic denitrification. Recent unpublished results indicate that combined aerobic nitrification/denitrification with methanotrophic/methylotrophic mixed cultures, growing on methane and on methanol produced as a by-product from methane, occurs under laboratory conditions (Mechsner, Kl., unpubl. results).

\section{Concluding remarks}

By no means does the foregoing discussion represent a comprehensive review of all questions concerning mixed substrates in environmental biotechnology. It simply provides a concise description of some appropriate recent work on the subject undertaken predominantly in the author's laboratory. There is a marked neglect of engineered environments other than waste treatment processes. In this context, a particularly pertinent example of sequential substrate utilization involves the offshore crude oil production industry.

The offshore production of crude oil has provided a major stimulus for the development of novel technologies that solve the numerous unique problems that such activities have generated. One development of major consequence in environmental biotechnology has been the development of an understanding of the mechanisms involved in hydrogen sulphide accumulation, and its prevention in offshore crude oil storage systems operating with seawater displacement. Both the problem and its solution have been discussed in detail by Wilkinson ${ }^{28}$. Other examples of the potential for environmental biotechnological analysis or evaluation of engineered structure operation can be expected to be identified. Such analyses and evaluations will result both in increased safety for personnel and in enhancement of the working life of engineered structures. Whilst such facets are unlikely to displace waste treatment process technology as biotechnology's primary involvement with environmental protection problems, they are likely to assume a much more pronounced rôle than they do at present.

Acknowledgements. Our thanks are due to the past and present doctoral candidates in our laboratory upon whose work much of this review is based and to the several funding agencies that made their work possible. In addition, we thank Mrs $\mathrm{R}$. Meisser for typing the manuscript and Mrs $H$. Bolliger for redrawing the figures.

1 Aebi, F., Eberli, W., Mörgeli, B., and von Rohr, Ph. R., Klärschlamm. Gas-Wasser-Abwasser 67 (1987) 102-110.

2 Al-Awadhi, N., The characterization and physiology of some thermotolerant and thermophilic solvent-utilizing bacteria. Doc. Diss. ETHZ No. 8810 (1989).

3 Bitzi, U., Abbau organischer Lösungsmittel mit bakteriellen Mischund Reinkulturen. Doc. Diss. ETHZ No. 8118 (1986).

4 Cripps, R. E., and Noble, A. S., The metabolism of nitrilotriacetate by a pseudomonad. Biochem. J. 136 (1973) 1059-1068. 
5 Dalton, H., Ammonia oxidation by the methane oxidising bacterium Methylococcus capsulatus strain bath. Archs Microbiol. 114 (1977) 273-279

6 Drozd, J. W., Bailey, M. L., and Godley, A., Oxidation of ammonia by methane-oxidizing bacteria. Proc. Soc. gen. Microbiol. 4 (1976) 26 .

7 Egli, Th., (An)aerobic breakdown of chelating agents used in household detergents. Microbiol. Sci. 5 (1988) 36-41.

$8 \mathrm{Egli}$, Th., Bosshard, Ch., and Hamer, G., Simultaneous utilization of methanol-glucose mixtures by Hansenula polymorpha in chemostat: influence of dilution rate and mixture composition on utilization pattern. Biotechnol. Bioeng. 28 (1986) 1735-1741.

9 Egli, Th., Weilenmann, H.-U., El-Banna, T., and Auling, G., Gramnegative, aerobic, nitrilotriacetate-utilizing bacteria from wastewater and soil. Syst. appl. Microbiol. 10 (1988) 297-305.

10 Gottschal, J.C., de Vries, S., and Kuenen, J. G., Competition between the facultatively chemolithotrophic Thiobacillus A2, an obligately chemolithotrophic Thiobacillus and a heterotrophic Spirillum for inorganic and organic substrates. Archs Microbiol. 121 (1979) $241-249$

11 Hamer, G., A biotechnological approach to the treatment of wastewater from petrochemicals manufacture. Instn chem. Engrs Symp. Ser. 77 (1983) 87-101.

12 Hamer, G., and Zwiefelhofer, H. P., Aerobic thermophilic hygienization - a supplement to anaerobic mesophilic waste sludge digestion. Chem. Engng Res. Des. 64 (1986) 417-424.

13 Humphrey, A. E., Moreira, A., Armiger, W., and Zabriskie, D., Production of single cell protein from cellulose wastes. Biotechnol. Bioeng. Symp. 7 (1977) 45-64.

14 Hutton, W. E., and ZoBell, C. E., Production of nitrite from ammonia by methane-oxidizing bacteria. J. Bact. 65 (1949) 216-219.

15 Leisinger, Th., Cook, A. M., Hütter, R., and Nüesch, J., (Eds) Microbial Degradation of Xenobiotic and Recalcitrant compounds. Academic Press, London 1981.

16 Linton, J. D., and Stephenson, R. J., A preliminary study on growth yields in relation to the carbon and energy content of varous organic growth substrates. FEMS Microbiol. Lett. 3 (1978) 95-98.

17 Mason, C. A., Microbial death, lysis and 'cryptic' growth: fundamental and applied aspects. Doc. Diss. ETHZ No. 8150 (1986).
18 Monod, J., Recherches sur la croissance des cultures bactériennes. Hermann, Paris 1942.

19 O'Neill, J. G., and Wilkinson, J. F., Oxidation of ammonia by methane-oxidizing bacteria and the effects of ammonia on methane oxidation. J. gen. Microbiol. 100 (1977) 407-412.

20 Prochazka, G. J., Payne, W. J., and Mayberry, W. R., Clorific contents of microorganisms. Biotechnol. Bioeng. 15 (1973) 1006-1011.

21 Robertson, L. A., and Kuenen, J. F., Thiospaera pantorropha gen. nov. sp. nov., a facultatively anaerobic, facultatively autotrophic sulphur bacterium. J. gen. Microbiol. 129 (1983) 2847-2855.

22 Robertson, L. A., and Kuenen, J. F., Aerobic denitrification: a controversy revived. Archs Microbiol. 139 (1984) 351-354.

23 Schneider, R. P., The NTA-monooxygenase from Pseudomonas sp. ATCC 29600. Doc. Diss. ETHZ No. 8824 (1989).

24 Senn, H. P., Kinetik und Regulation des Zuckerabbaus von Escherichia coli ML 30 bei tiefen Zuckerkonzentrationen. Doc. Diss. ETHZ No. 8831 (1989).

25 Stumm-Zollinger, E., Substrate utilization in heterogeneous bacterial communities. J. Wat. Pollut. Control Fed. 40 (1968) R213-R229.

26 Wehrli, E., and Egli, Th., Morphology of nitrilotriacetate-utilizing bacteria. Syst. appl. Microbiol. 10 (1986) 306-312.

27 Wilkinson, T. G., Interactions in a mixed bacterial population growing on methane in continuous culture. Doc. Diss. Univ. London (1972).

28 Wilkinson, T. G., An environmental programm for offshore oil operations. Chem. Indust. (1982) 115-123.

29 Wilkinson, T. G., and Hamer, G., The microbial oxidation of mixtures of methanol, phenol, acetone and isopropanol with reference to effluent purification. J. chem. Technol. Biotechnol. 29 (1979) 56-67.

30 Wilkinson, T. G., Topiwala, H. H., and Hamer, G., Interactions in a mixed bacterial population growing on methane in continuous culture. Biotechnol. Bioeng. 16 (1974) 41-59.

31 Zlokarnik, M., Bioengineering aspects of aerobic waste water purification. Ger. Chem. Engng. 6 (1983) 183-197.

$0014-4754 / 89 / 11-12 / 1070-09 \$ 1.50+0.20 / 0$

(C) Birkhäuser Verlag Basel, 1989 